Communications in Physics, Vol. 24, No. 3S2 (2014), pp. 107-118

DOI:10.15625/0868-3166/24/3S2/5056

\title{
DESIGN AND DEVELOPMENT OF THE FOLDED 4-MIRROR RESONATORS FOR DIODE END-PUMPED SOLID-STATE CR:LISAF LASERS
}

\author{
NGUYEN VAN HAO \\ Institute of Physics, Vietnam Academy of Science and Technology \\ and \\ Thai Nguyen University of Science, Thai Nguyen City, Vietnam \\ PHAM VAN DUONG, PHAM HONG MINH, DO QUOC KHANH \\ Institute of Physics, Vietnam Academy of Science and Technology \\ ANTONIO AGNESI \\ Laser Source Laboratory, Department of Electrical, \\ Computer and Biomedical Engineering, University of Pavia. Italy \\ E-mail: nvhao@grad.iop.vast.ac.vn \\ Received 04 April 2014 \\ Accepted for publication 24 May 2014
}

\begin{abstract}
We present the design and development of folded four-mirror resonators for diode end-pumped solid-state Cr:LiSAF lasers. The astigmatic effects due to the Brewster-cut laser crystal and two curved mirrors used at oblique incidence were taken into consideration. The obtained results showed the stability regions of resonator, the beam parameters in the resonator and within the laser crystal as well as other sensitive parameters and their variation ranges. Furthermore, the designed folded four-mirror resonators for diode end-pumped Cr:LiSAF laser were experimentally evaluated. The CW Cr:LiSAF laser characteristics in threshold, efficiency and beam quality as well as comparative studies are presented.
\end{abstract}

Keywords: Optical resonator, laser diode pumping, Cr:LiSAF laser crystal.

\section{INTRODUCTION}

Diode-pumped, broadly tunable and solid-state laser sources are attractive for many scientific and technological applications [1]. Development of such lasers requires cavity designs to provide useful information about the stability regions of the cavity, the beam parameters in the cavity and within the laser crystal, saturable absorber... Among the diode-pumped solid-state laser media, the Cr:LiSAF has the broad gain bandwidth that supports laser emission continuously tunable from 750 to $1000 \mathrm{~nm}$ [2-9]. As a result of its absorption band red shifted to $\sim 670 \mathrm{~nm}$, one $\mathrm{Cr}$ :LiSAF enables direct diode pumping with low-cost diode lasers, significantly reduces the total cost of the diode-pump laser system and beam quality depend strongly on the design of laser resonator. Beside, the optimization of pumping power that is focused on the laser crystal, optimally

(C)2014 Vietnam Academy of Science and Technology 
designed resonator is required to achieve an extremely good mode matching (spatial overlap) between the pump beam and laser beam inside laser crystal $[6,10-16]$. Therefore, the development of diode - pumped solid state lasers requires precise designs of laser resonator.

In this work, we present the designs for the folded four-mirror resonators of diode endpumped Cr:LiSAF lasers using the $A B C D$ formalism for intra-cavity Gaussian beam. The astigmatic effects due to the Brewster laser crystal and two curved mirrors used at oblique incidence were taken into this consideration. In the case, compensation of the astigmatic effects is based on the use of another source of astigmatism to be off-axis reflection from the curved mirrors into the laser crystal. The obtained results showed the stability regions of resonator, the beam parameters in the resonator and within the laser crystal as well as any other sensitive parameters and their variation ranges. Furthermore, the designed folded four-mirror resonators for diode end-pumped Cr:LiSAF lasers were experimentally evaluated. We found that the Cr:LiSAF lasers successfully operated with a threshold as low as $11.3 \mathrm{~mW}$, a slope efficiency as high as $35.6 \%$ and a fundamental mode $\left(\mathrm{TEM}_{00}\right)$. These laser characteristics are a little better than those obtained recently by other groups.

\section{DESIGN OF Cr:LiSAF LASER RESONATOR}

Our aim is to design the folded resonators to develop diode end-pumped CW Cr:LiSAF laser and diode end pumped mode-locked Cr:LiSAF laser. These resonators satisfy the requirement of long length and tight focusing with internal lenses or, equivalently, resonator with folded arms, therefore, a folded four -mirror resonator configuration is chose (Fig. 1). The resonator consists of two flat mirror $M_{3}, M_{4}$ and two curved mirrors $M_{1}, M_{2}$. One of the flat mirrors $\left(M_{3}\right.$, $\left.M_{4}\right)$ is a high reflector mirror and another is an output coupler. Two curved mirrors $\left(M_{1}, M_{2}\right)$ mainly focus the fluorescence and laser emission into the laser crystal, these mirrors are of very high reflectivity at laser wavelength and a very low reflectivity at pump diode wavelength. Laser resonator must ensure an extremely good mode matching between the pump diode beam and laser beam inside the laser crystal $[13,14,17]$.

In the technology of solid-state lasers, Brewster-cut laser crystals have been widely used to avoid mode parasite and intra-cavity losses. However, such an intra-cavity Brewster-cut laser crystal that can cause the effect of asymmetric producing astigmatic Gaussian beams. The beams have different spot sizes, wave-front curvatures and beam waist positions in the two orthogonal planes called the sagittal plane and the tangential plane (Fig. 1). In other word, because of a lack of axial symmetry in the resonator, the beam waist along the sagittal and tangential planes may not overlap spatially due to astigmatism. There are two focusing lengths of the laser crystal depending upon the plane in which the beam is focused $[13,14,17]$.

$$
f_{t}=f \cos \alpha \text { and } f_{s}=\frac{f}{\cos \alpha}
$$

where $f_{s, t}$ are the focal lengths in the sagittal and tangential planes, $f=R / 2$ is the on-axis focal length of the mirror and $\alpha$ is the angle between the incident beam and the normal to the mirror.

An intra-cavity Brewster-cut laser crystal also has essentially two different effective optical lengths in the sagittal and tangential planes [14]. The effective lengths in the two different planes for a Brewster-cut crystal of the length $l$ and refractive index $n$ are given by $[14,15]$ :

$$
l_{S}=l / n \text { and } l_{t}=l / n^{3}
$$


where $l_{s, t}$ are the effective lengths in the sagittal and tangential planes.

These astigmatic effects must be taken into account in cavity stability analysis. Compensation of astigmatism is necessary to produce a maximum overlap of the stability regimes and the designed laser mode characteristics. In our consideration, this can be achieved by the combination of a Brewster-cut crystal and an inclined curved mirror with a common plane of incidence in the same resonator. In the case, compensation of these effects is to use another source of astigmatism such as off-axis reflection from the curved mirrors into the laser crystal, as shown in Fig. 1.

The resonator configuration of our diode-pumped Cr:LiSAF laser is shown in Fig. 1. The Cr:LiSAF crystal that we used as a gain medium is a 3\%-doped, 4-mm-long, Brewster-cut, Cr:LiSAF crystal (VLOC, Inc). $M_{1}$ and $M_{2}$ are curved and high reflection mirrors from 780 to $900 \mathrm{~nm}$ $(R>99.9 \%)$ and $>95 \%$ transmission at the pump wavelength, they have the curvature radii of $50 \mathrm{~mm}$ and $100 \mathrm{~mm}$, respectively. $M_{3}$ and $M_{4}$ are flat mirrors, $M_{3}$ is a high reflector mirror from 780 to $900 \mathrm{~nm}(R>99.9 \%)$ and $M_{4}$ is used as an output coupler.

The Cr:LiSAF laser crystal is pumped at $\sim 660 \mathrm{~nm}$ by a linearly-polarized, AlGaInP singlemode diode (Mitsubishi, 101J27- 01) with a maximum pump power of $120 \mathrm{~mW}$. The output of the diode beam was collimated by aspheric lenses $(f=6 \mathrm{~mm})$. One $50-\mathrm{mm}$ focal length lens focuses the pump beams in the Cr:LiSAF crystal. A pump beam waist radius of about $15 \mu \mathrm{m}$ inside the Cr:LiSAF crystal could be achieved and measured. We have to design the resonator to obtain laser mode size of about $15 \mu \mathrm{m}$ inside the Cr:LiSAF crystal. This is necessary condition to achieve a good mode matching between the pump beam and laser beam inside the laser crystal.

a)

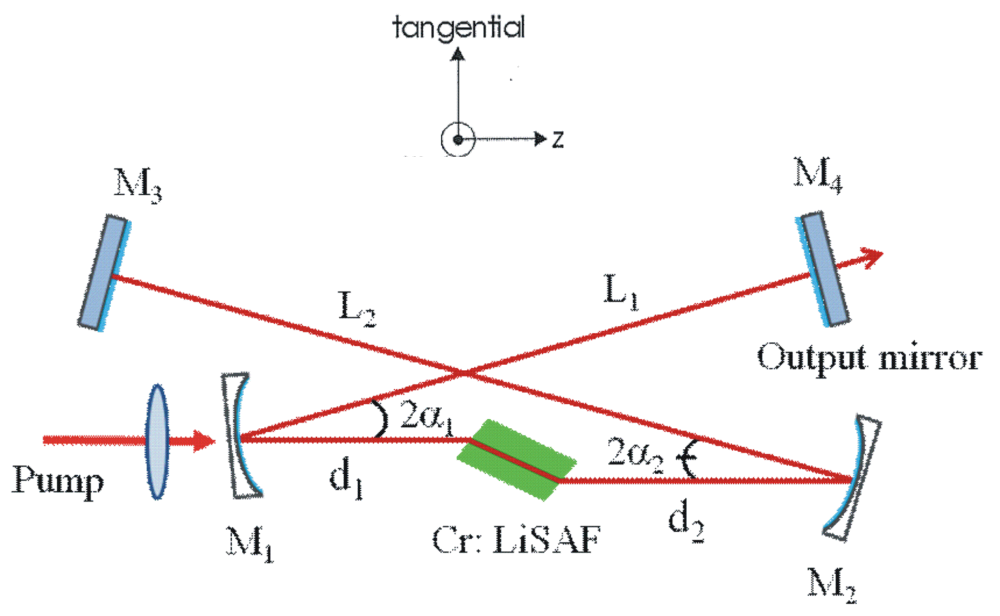

b)

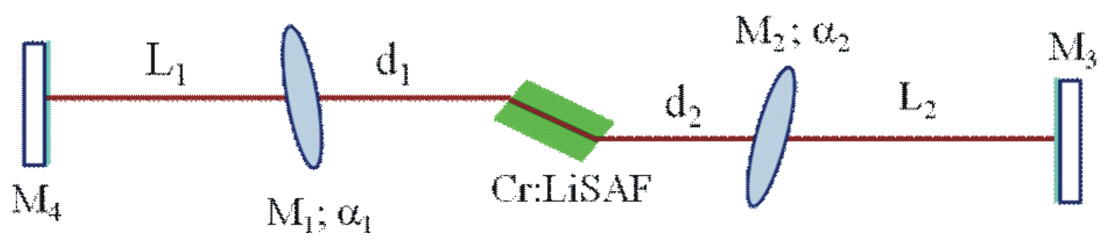

Fig. 1. (a) The $\mathrm{X}$-folded 4-mirror Cr:LiSAF laser resonator; $d_{1}, d_{2}, L_{1}$ and $L_{2}$ were defined (b) it's equivalent resonator. The gain medium is a Brewster-cut Cr:LiSAF crystal; $M_{1}$ and $M_{2}$ are curved mirrors; $M_{3}$ and $M_{4}$ are flat mirrors. 
Numeric calculations were carried out with use of the complex Gaussian beam parameter $q$ and the $A B C D$ matrix of each of the resonator elements in sequence. The complex Gaussian beam parameter $q$ at one plane with position $z$ in this resonator is determined by $[14,16]]$ :

$$
\frac{1}{q_{\text {out }}}=\frac{C+\left(D / q_{\text {in }}\right)}{A+\left(B / q_{\text {in }}\right)} \text { and } \frac{1}{q(z)}=\frac{1}{R(z)}-\frac{i \lambda}{\pi \omega^{2}(z)}
$$

where $R(z)$ is the wavefront radius of curvature and $\omega(z)$ is the beam radius at position $z$. At any point in the optical system, this complex beam radius can be obtained with the $A B C D$ transfer matrix. $A, B, C$, and $D$ are the components of the $A B C D$ matrix of the optical elements between the input and the arbitrary output points.

Complex beam parameter in one round-trip from the mirror $M_{4}$ position can be determined from the $A B C D_{1}$ matrix ( $M_{4}$ is the reference plane):

$$
A B C D_{1}=M_{L_{2}} M_{M_{2}} M_{d_{2}} M_{C r} M_{d_{1}} M_{M_{1}} M_{L_{1}}=\left[\begin{array}{cc}
A_{1} & B_{1} \\
C_{1} & D_{1}
\end{array}\right]
$$

When the laser beam propagates in opposite direction, we have the following $\mathrm{ABCD}_{2}$ matrix:

$$
A B C D_{2}=\left[\begin{array}{ll}
D_{1} & B_{1} \\
C_{1} & A_{1}
\end{array}\right]
$$

and when the laser beam propagates a full cavity round-trip, we have $\mathrm{ABCD}$ matrix, with

$$
A B C D=A B C D_{2} \cdot A B C D_{1}
$$

Stable condition for laser resonator is:

$$
|(A+D) / 2| \leq 1
$$

where $(A+D)$ is the trace of the matrix (6).

The beam radius, $\omega$ which is given by relation $[14,16]$ :

$$
\omega^{2}(z)=\frac{\lambda|B|}{\pi} \sqrt{\frac{1}{1-\frac{(A+D)^{2}}{4}}}
$$

Because of astigmatic effects, the complex Gaussian beam parameter $q$, the $A B C D$ matrix, the stability range and the beam parameters are different in the tangential and sagittal planes. Therefore, computations are made separately in the two planes, and the overlapped region is indentified as the stability region of the resonator. A typical result is presented in Fig. 2. Solid and dashed curves indicate the tangential and sagittal planes, respectively.

Fig. 2 shows the stability ranges for $d_{1}$ and $d_{2}$ for the folded 4-mirror resonator of the Cr:LiSAF laser as shown in Fig. 1 . We see that each value of $d_{1}$ can have two stability ranges of $d_{2}$.

Fig. 3 shows the stability ranges for $\alpha_{1}$ and $\alpha_{2}$ of the folded 4-mirror resonator of the Cr:LiSAF laser operating in the first stability range. Allowable values of $\alpha_{1}$ and $\alpha_{2}$ are rather large, $\alpha_{1}$ can only change from $6^{\circ}$ to $13^{\circ}$ while $\alpha_{2}$ from $5^{\circ}$ to $10^{\circ}$. We can see that a beam waist radius (laser mode) at position of the laser crystal is about $14 \mu \mathrm{m}$, corresponding to $\alpha_{1}=7.5^{\circ}$ and $\alpha_{2}=6^{\circ}$.

Similarly, the stability ranges for $\alpha_{1}$ and $\alpha_{2}$ of the folded 4-mirror Cr:LiSAF laser resonators operating in the second stability range were carried out. Changeable values of $\alpha_{1}$ and $\alpha_{2}$ 


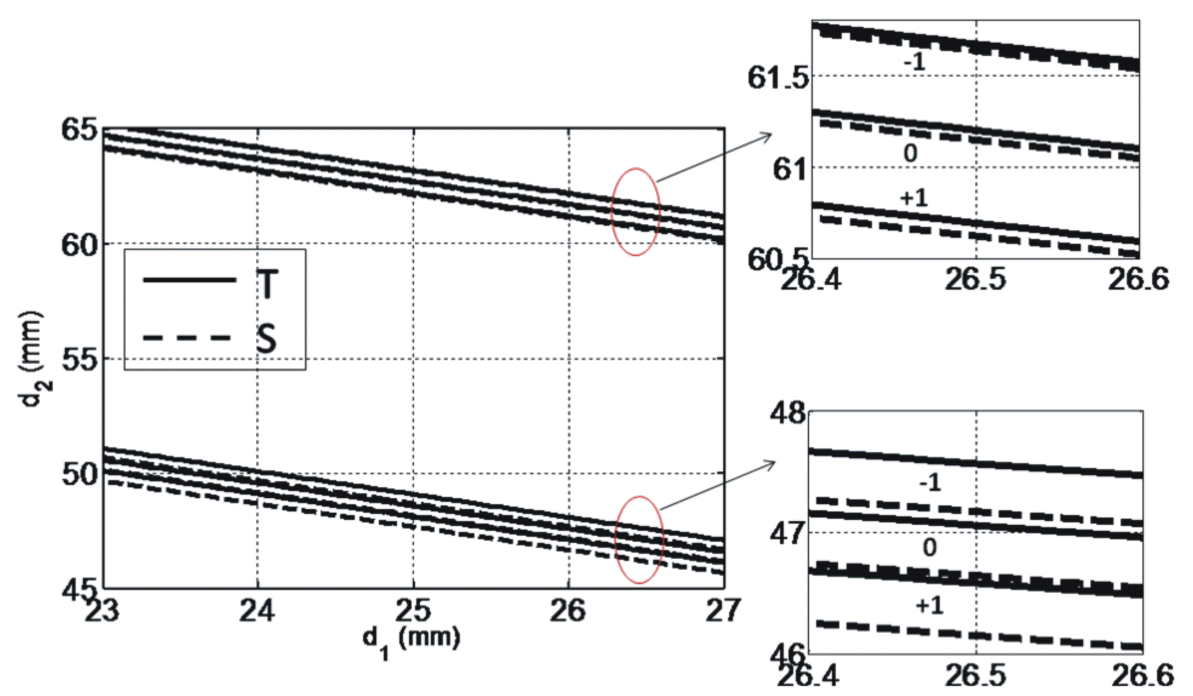

Fig. 2. Stability ranges for $\mathrm{d}_{1}$ and $\mathrm{d}_{2}$ of the folded four-mirror Cr: LiSAF laser resonator shown in Fig. $1\left(\alpha_{1}=7.5^{\circ}, \alpha_{2}=6^{\circ}, L_{1}=430 \mathrm{~mm} ; L_{2}=300 \mathrm{~mm}\right.$ and $\left.\lambda=870 \mathrm{~nm}\right)$. Solid and dashed lines indicate the tangential and sagittal planes, respectively.
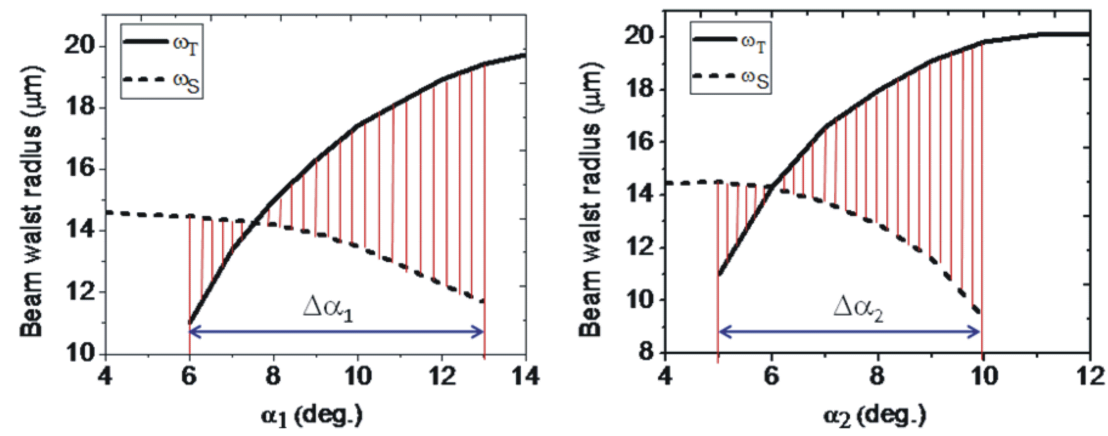

Fig. 3. Stability ranges for variation of $\alpha_{1}\left(\alpha_{2}=6^{\circ}\right)$ and $\alpha_{2}\left(\alpha_{1}=7.5^{\circ}\right)$ in the sagittal and tangential planes for the folded 4 - mirror resonator shown in Fig. $1\left(d_{1}=24.25 \mathrm{~mm}\right.$, $d_{2}=48.95 \mathrm{~mm}, L_{1}=430 \mathrm{~mm}, L_{2}=300 \mathrm{~mm}$ and $\lambda=870 \mathrm{~nm}$ ). A beam waist radius at position of the laser crystal is about $14 \mu \mathrm{m}$.

are rather large, $\alpha_{1}$ and $\alpha_{2}$ can change in the intervals of $5^{\circ}$ and $7^{\circ}$, respectively. The results showed a beam waist radius at position of the laser crystal to be about $14.2 \mu \mathrm{m}$ when $\alpha_{1}=7.5^{\circ}$ and $\alpha_{2}=6^{\circ}$. This size of laser mode is well suitable to that of the pump mode (14 $\left.\mu \mathrm{m}\right)$. For comparative studies, the parameters $\alpha_{1}=7.5^{\circ}$ and $\alpha_{2}=6^{\circ}$ were used in the calculations of the folded 4-mirror resonator for the Cr:LiSAF lasers operating in two stability ranges. 


\section{About the first stability range:}

Fig. 4 shows the first stability range for $d_{1}$ and $d_{2}$ of the folded 4-mirror Cr: LiSAF laser resonator in the sagittal and tangential planes. Allowable values of $d_{1}$ and $d_{2}$ are in a very small interval of $\sim 1 \mathrm{~mm}, d_{1}$ can only change from 24.17 to $25.1 \mathrm{~mm}$ while $d_{2}$ from 48.86 to $49.75 \mathrm{~mm}$. We can say $d_{1}$ and $d_{2}$ are sensitive cavity parameters, the positions of two spherical mirrors $\left(M_{1}\right.$ and $M_{2}$ ) are very sensitive to the stability of the Cr:LiSAF laser resonators.
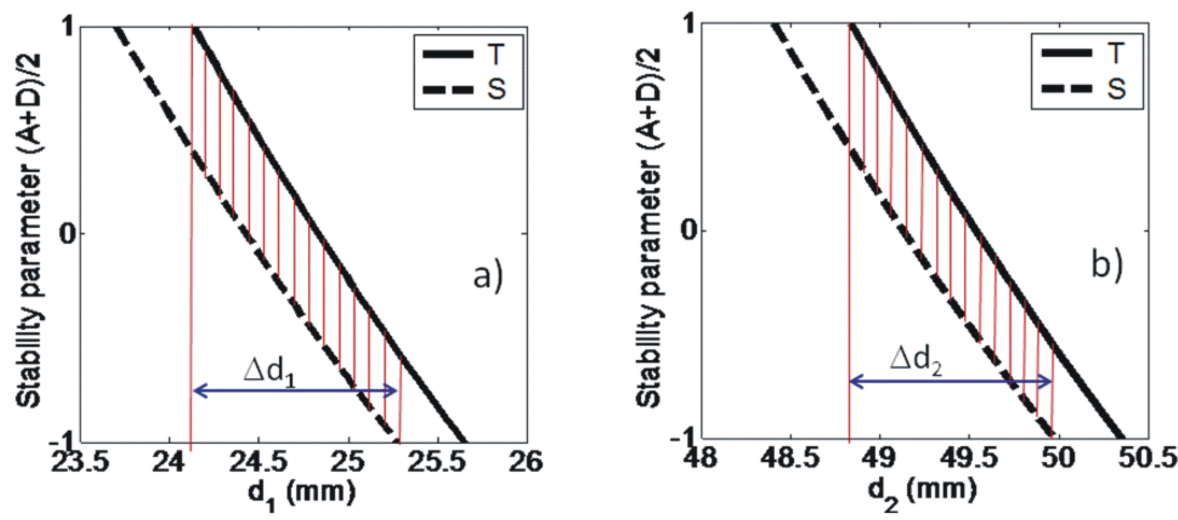

Fig. 4. Stability range for $d_{1}\left(d_{2}=48.95 \mathrm{~mm}\right)$ and $d_{2}\left(d_{1}=24.25 \mathrm{~mm}\right)$ of the Cr:LiSAF laser resonator as shown in Fig. $1\left(\alpha_{1}=7.5^{\circ}, \alpha_{2}=6^{\circ}, L_{1}=430 \mathrm{~mm} ; L_{2}=300 \mathrm{~mm}\right.$ and $\lambda=870 \mathrm{~nm})$.

Fig. 5 shows the stability range for $L_{1}$ and $L_{2}$ of the folded 4-mirror Cr: LiSAF laser resonator in the sagittal and tangential planes. $L_{1}$ and $L_{2}$ can change in a wide range, however, when $L_{1}$ and $L_{2}$ are large, the mismatching of the laser beam in the sagittal and tangential planes are larger (Fig. 6). This feature should be considered carefully into the design, alignment and application of the laser.
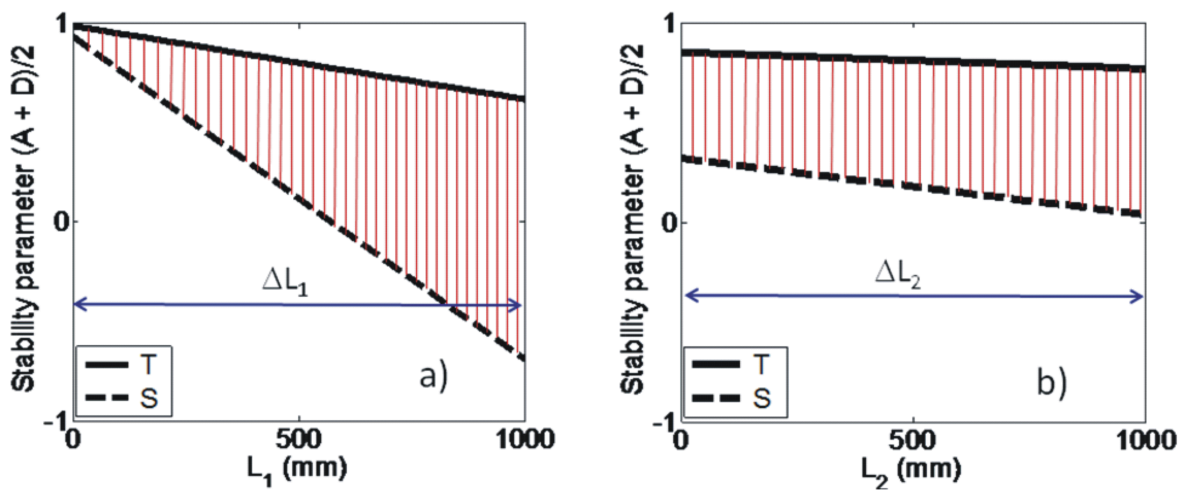

Fig. 5. Stability ranges for $L_{1}\left(L_{2}=300 \mathrm{~mm}\right)$ and $L_{2}\left(L_{1}=430 \mathrm{~mm}\right)$ of the Cr:LiSAF laser resonator shown in Fig. $1\left(d_{1}=24.25 \mathrm{~mm}, d_{2}=48.95 \mathrm{~mm}, \alpha_{1}=7.5^{\circ}, \alpha_{2}=6^{\circ}\right.$, and $\lambda=870 \mathrm{~nm}$ ). 
Fig. 6 presents laser beam radius versus position for the folded 4-mirror resonator in the tangential and sagittal planes. It is noted that astigmatism is well eliminated within the laser crystal but not in $M_{3}$ and $M_{4}$, i.e., the beam waist in sagittal plane is not equal to the beam waist in the tangential plane. Fig. 6 shows the minimal beam waist of the resonator at position of the laser crystal to be $\sim 14.2 \mu \mathrm{m}$. Such a resonator configuration can achieve a good mode matching between the pump beam and laser beam inside laser crystal, therefore, a high laser efficiency and a low pump threshold.

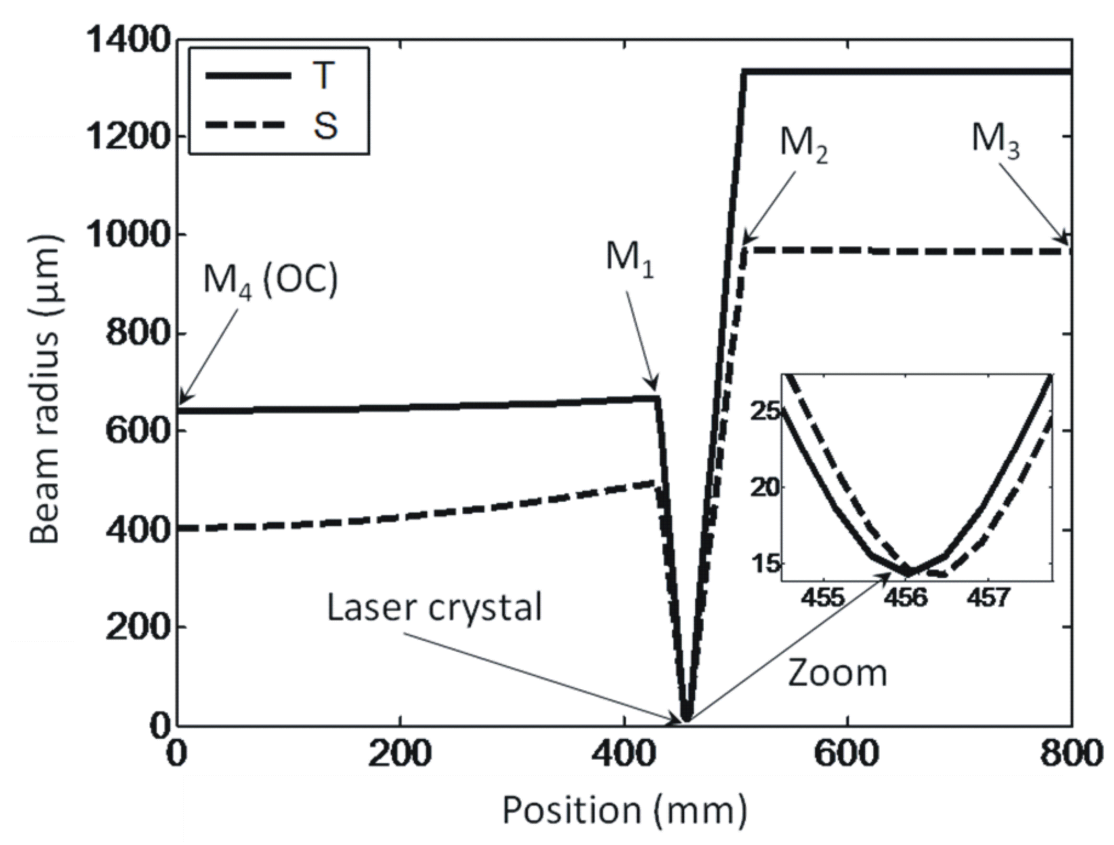

Fig. 6. Laser beam waist radius versus position for the folded 4-mirror resonator as shown in Fig. $1\left(d_{1}=24.25 \mathrm{~mm}, d_{2}=48.95 \mathrm{~mm}, \alpha_{1}=7.5^{\circ}, \alpha_{2}=6^{\circ}, L_{1}=430 \mathrm{~mm}\right.$; $L_{2}=300 \mathrm{~mm}$ and $\lambda=870 \mathrm{~nm}$ ).

\section{About the second stability range:}

The same calculations have been done for the folded 4-mirror diode end-pumped CW Cr:LiSAF laser that operated in the second stability range. Similarly, the parameters $\alpha_{1}=7.5^{\circ}$ and $\alpha_{2}=6^{\circ}$ were used in the calculations for the CW Cr:LiSAF lasers operating in the second stability range.

Fig. 7 shows the second stability ranges for $d_{1}$ and $d_{2}$ of the folded 4-mirror Cr: LiSAF laser resonator in the sagittal and tangential planes. Changeable values of $d_{1}$ and $d_{2}$ are in a small interval of about $1 \mathrm{~mm}, d_{1}$ can only change from 24.17 to $25.15 \mathrm{~mm}$ while $d_{2}$ from 62.1 to 63.05 mm. $d_{1}$ and $d_{2}$ are sensitive cavity parameters, the positions of two spherical mirrors $\left(M_{1}\right.$ and $\left.M_{2}\right)$ are very sensitive for the stability of the folded 4-mirror Cr:LiSAF laser resonators.

Fig. 8 shows the stability ranges for $\mathrm{L}_{1}$ and $\mathrm{L}_{2}$ of the folded 4-mirror $\mathrm{Cr}$ : LiSAF laser resonator. $\mathrm{L}_{1}$ and $\mathrm{L}_{2}$ can change in a wide range, while the matching of the laser beam in the 

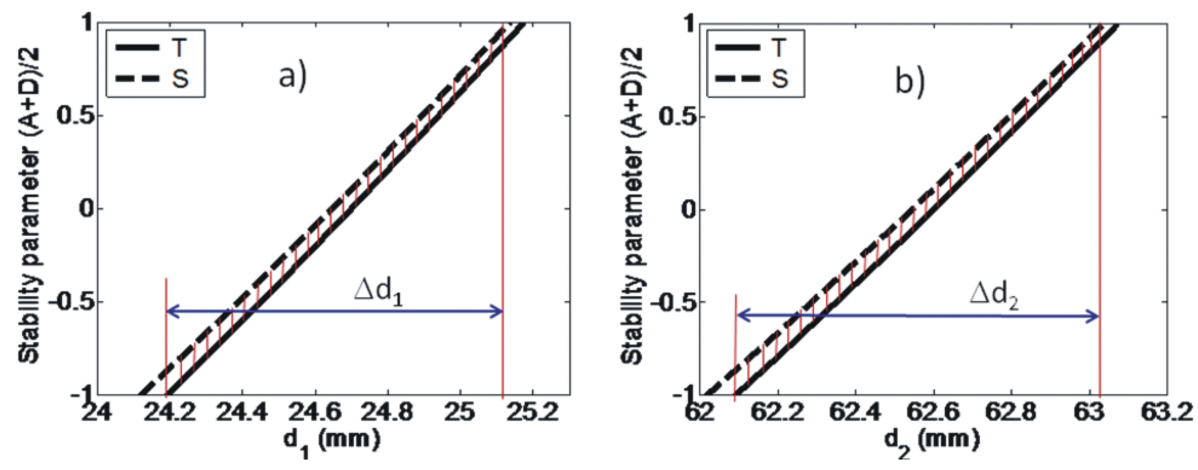

Fig. 7. Stability ranges for $d_{1}\left(d_{2}=63 \mathrm{~mm}\right)$ and $d_{2}\left(d_{1}=25.1 \mathrm{~mm}\right)$ of the Cr:LiSAF laser resonators $\left(\alpha_{1}=7.5^{\circ}, \alpha_{2}=6^{\circ}, L_{1}=650 \mathrm{~mm} ; L_{2}=220 \mathrm{~mm}\right.$ and $\left.\lambda=870 \mathrm{~nm}\right)$.
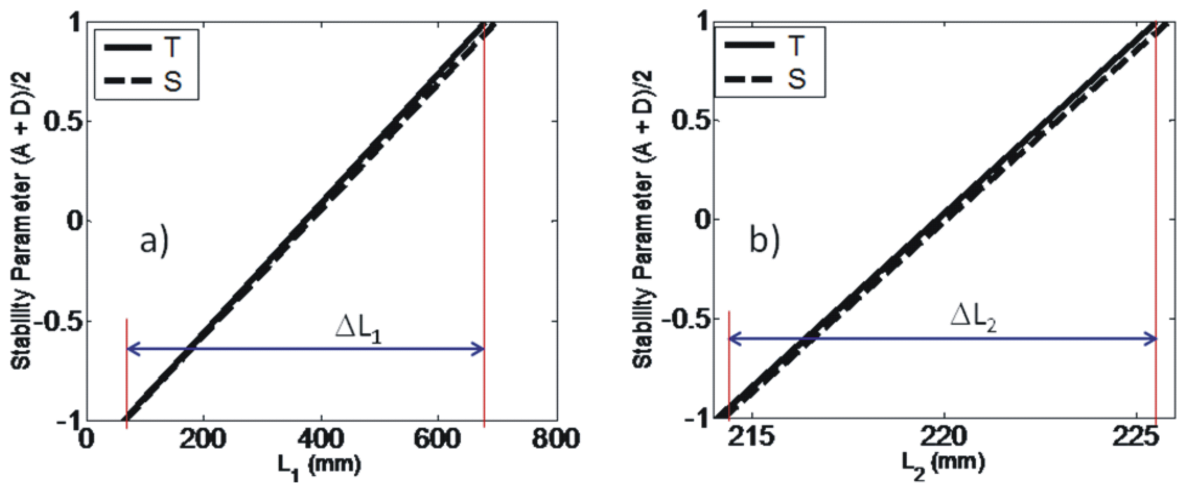

Fig. 8. Stability ranges for $L_{1}\left(L_{2}=220 \mathrm{~mm}\right)$ and $\mathrm{L}_{2}\left(\mathrm{~L}_{1}=650 \mathrm{~mm}\right)$ of the Cr:LiSAF laser resonator $\left(\mathrm{d}_{1}=25.1 \mathrm{~mm}, \mathrm{~d}_{2}=63 \mathrm{~mm}, \alpha_{1}=7.5^{\circ}, \alpha_{2}=6^{\circ}\right.$, and $\left.\lambda=870 \mathrm{~nm}\right)$.

sagittal and tangential planes is much better (Fig. 9) than that of the laser operating in the first stability range (Fig. 6).

Fig. 9 shows laser beam radius versus position for the folded 4-mirror diode end-pumped Cr:LiSAF laser (Fig.1) in both the sagittal and tangential planes. Astigmatic compensation in this resonator is realized much better than that of the laser resonators operating in the first stability range (Fig.6). Astigmatism is eliminated not only within the laser crystal but also in $M_{3}$ and $M_{4}$. The graph shows the minimal beam waist is $10.5 \mu \mathrm{m}$ at the position of the laser crystal. In addition, the second folded arm has a focus of a beam waist radius $\sim 40 \mu \mathrm{m}$ (Fig. 9 right). This position is very favorable to insert a SESAM for a mode-locked laser operation.

\section{EXPERIMENT AND DISCUSSION}

The designed folded four-mirror resonators of diode end-pumped Cr:LiSAF laser have been experimentally evaluated.

The schematic of the folded 4-mirror diode end-pumped Cr:LiSAF laser operated in the first stability range is presented in Fig. 10a. The laser used a folded four-mirror resonator of the 


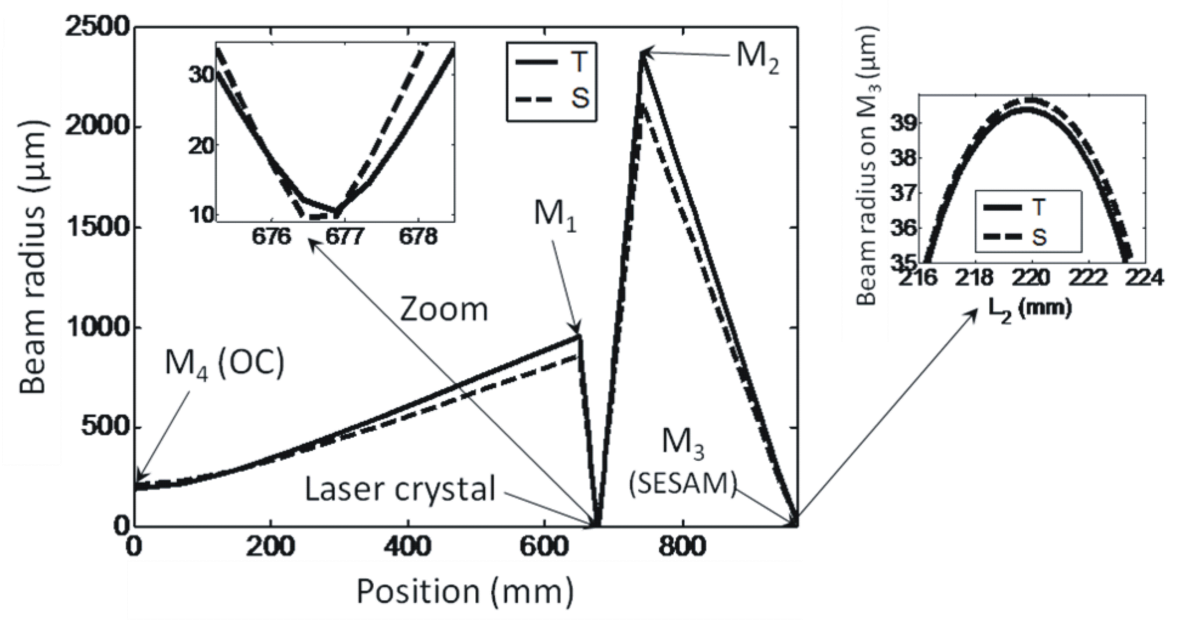

Fig. 9. Beam waist radius versus position for the Cr:LiSAF resonator as shown in Fig.1 $\left(\mathrm{d}_{1}=25.1 \mathrm{~mm}, \mathrm{~d}_{2}=63 \mathrm{~mm}, \alpha_{1}=7.5^{\circ}, \alpha_{2}=6^{\circ}, \mathrm{L}_{1}=650 \mathrm{~mm} ; \mathrm{L}_{2}=220 \mathrm{~mm}\right.$ and $\lambda=$ $870 \mathrm{~nm})$.

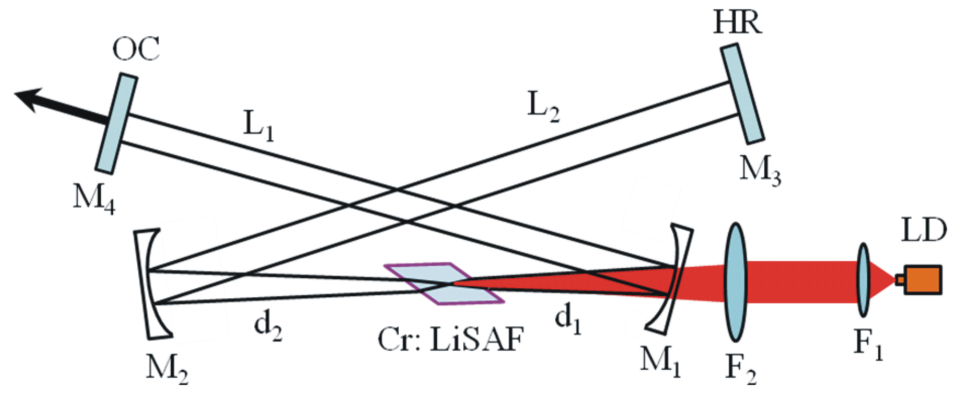

Fig. 10. Folded 4-mirror Cr:LiSAF laser resonator developed in the first stability range $\left(\mathrm{L}_{1}=430 \mathrm{~mm}\right.$ and $\mathrm{L}_{2}=300 \mathrm{~mm}, \mathrm{~d}_{1}=24.25 \mathrm{~mm}$ and $d_{2}=48.95 \mathrm{~mm}, \alpha_{1}=7.5^{\circ}, \alpha_{2}=$ $\left.6^{\circ}\right) . M_{1}$ and $M_{2}$ have curvature radii of $R_{1}=-50 \mathrm{~mm} R_{2}=-100 \mathrm{~mm}$, respectively. $M_{3}$ and $M_{4}$ are flat mirrors; The gain medium is a Brewster-cut Cr:LiSAF crystal of length $l=4 \mathrm{~mm}$ and refractive index $n=1.41$.

arm lengths $\left(L_{1}=430 \mathrm{~mm}\right.$ and $L_{2}=300 \mathrm{~mm}, d_{1}=24.25 \mathrm{~mm}$ and $\left.d_{2}=48.95 \mathrm{~mm}\right)$ in order to obtain a beam waist of $\sim 14.2 \mu$ m inside the laser crystal (Fig. 6).

The schematic of the folded 4-mirror diode end-pumped Cr:LiSAF laser operated in the second stability range, is presented in Fig. 10b. The laser used a folded four-mirror resonator of the arm lengths $\left(L_{1}=650 \mathrm{~mm}\right.$ and $L_{2}=220 \mathrm{~mm}, d_{1}=25.1 \mathrm{~mm}$ and $\left.d_{2}=63 \mathrm{~mm}\right)$ to obtain a beam waist of $\sim 11 \mu \mathrm{m}$ inside the laser crystal and a beam waist of $\sim 40 \mu \mathrm{m}$ on the mirror $M_{3}$ (Fig. 9). 
Laser energy was measured by the Power meters (Ophir NOVA-OP, Newport and 13 PEM001 Melles Griot). The laser wavelength and spectra were recorded with a grating spectrometer (DFS8, Russia) and an Optical Spectrum Analyzer (Ando AQ6317B, Japan) coupling with a singlemode optical fiber. Laser beam quality and size were evaluated with knife-edge techniques.

Fig. 11 shows powerful characteristics of the folded 4-mirror diode-pumped, CW Cr:LiSAF laser operated in the first stability range using several different OC transmissions $(0.46 \% ; 1 \%, 2$ $\%$ and $5 \%$ ). Thresholds as low as $11.3 \mathrm{~mW}$ were measured with the $0.46 \%$ output coupler. The highest $\mathrm{CW}$ output power $(31.8 \mathrm{~mW})$, a $31.5 \mathrm{~mW}$ lasing threshold and $35.6 \%$ slope efficiency were obtained using the $2 \%$ output coupler. The laser characteristics are quite comparable with those reported to date [6-9].

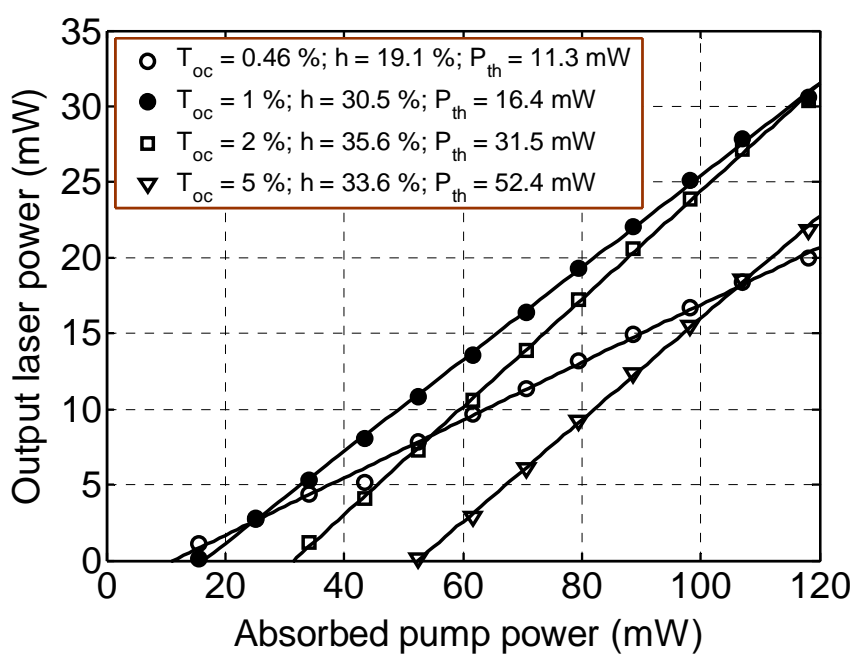

Fig. 11. Powerful characteristics of the diode-pumped, CW Cr:LiSAF laser operated with several different OC transmissions $(0.46 \%, 1 \%, 2 \%$ and $5 \%)$.

Using the measured pump powers at threshold for several different output couplers with low transmission $(0.46 \%, 1 \%$ and $2 \%)$ a roundtrip cavity loss of $0.94 \%$ was estimated with Findlay-Clay calculations [18]. This value is in good agreement with the mirror reflectivities and the low scatering losses inside the laser crystal and comparable to that reported to date [7-9].

Fig. 12 presents optical spectrum of the folded 4-mirror diode end-pumped CW Cr:LiSAF laser emission with the output coupler of $1 \%$ transmission at a pump power of $99 \mathrm{~mW}$. For the pump diode power at 7 times above threshold, a spectral bandwidth of $1.8 \mathrm{~nm}$ centered at $869 \mathrm{~nm}$ was measured. Such a laser bandwidth value is narrow due to the limit in pump diode power.

Fig. 13 shows the measured results of beam waist radius of the folded 4-mirror CW Cr:LiSAF laser in both $\mathrm{x}$-axis and $\mathrm{y}$-axis. We found that Cr:LiSAF laser operate in fundamental mode $\left(\mathrm{TEM}_{00}\right)$.

The same characterizations were done for the folded 4-mirror diode end-pumped CW Cr:LiSAF laser that operating in the second stability range. The experimental results showed that the characteristics of this Cr:LiSAF laser are better than those of the CW Cr:LiSAF laser in 


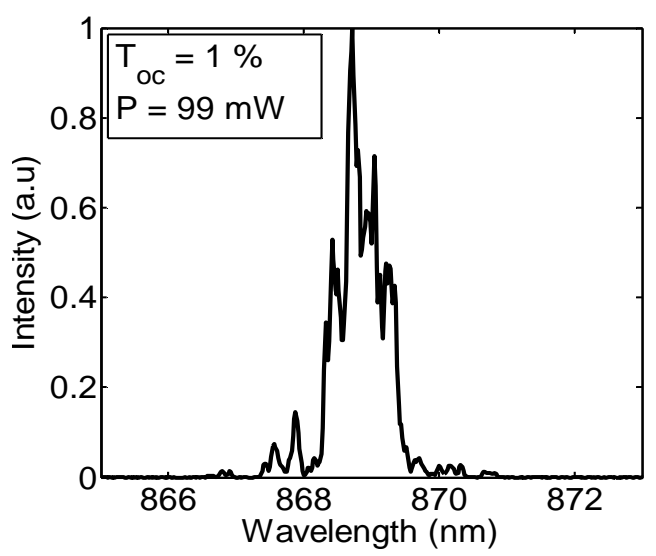

Fig. 12. Optical spectrum of the diode pumped CW Cr:LiSAF laser with OC transmission of 1 $\%$ at pump power of $99 \mathrm{~mW}$.

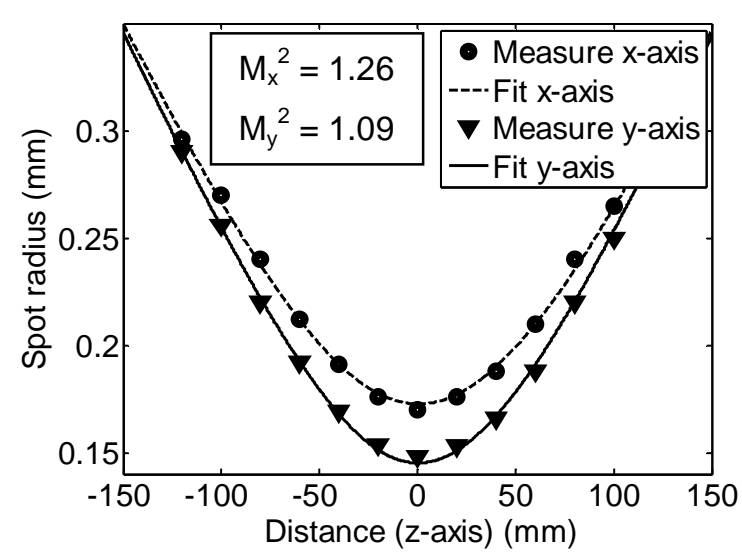

Fig. 13. Beam waist radius of $\mathrm{CW} \mathrm{Cr}$ : LiSAF laser in both $\mathrm{x}$-axis and $\mathrm{y}$-axis. Cr:LiSAF laser operating in fundamental mode with good beam quality.

the first stability range. This can be understood as follows: firstly, the Cr:LiSAF lasers used the stable folded four-mirror resonator, the same laser and optical elements and pump laser diode; secondly, this laser resonator has three advantages over that in the first stability range such as a longer cavity length, two intra-cavity focuses and better elimination of astigmatic effects. Such a laser resonator configuration is quite suitable to diode end-pumped mode-locked Cr:LiSAF lasers. The characteristics of this folded 4-mirror Cr:LiSAF laser in the second stability range will be presented more detail in future publication.

\section{CONCLUSION}

In conclusion, two folded 4-mirror resonator configurations for diode end-pumped Cr:LiSAF lasers have been numerically designed and evaluated experimentally. We have showed clearly the stability regions of the resonator, astigmatic compensation, the beam parameters inside the resonator and within the laser crystal, or a SESAM as well as other sensitive parameters $\left(\mathrm{d}_{1}\right.$ and $\left.\mathrm{d}_{2}\right)$ and their variation ranges. There are useful in the design, alignment and development of folded 4-mirror resonators for diode end-pumped solid-state laser. The folded 4-mirror laser resonator developed in the second stability range has more advantages than that in the first stability range. Such a laser resonator configuration is suitable to diode end -pumped mode-locked Cr:LiSAF lasers.

\section{ACKNOWLEDGMENT}

The authors would like to thank for the financial support from Vietnam NAFOSTED (Physics, Project $\mathrm{N}^{0}$. 103.06.89.09).

\section{REFERENCES}

[1] Ursula Keller, Nature 424 (2003) 831-837.

[2] Richard Scheps, Introduction to Laser Diode-pumped Solid State Lasers. SPIE - The International Society of Optical Engineering, 2002. 
[3] S. A. Payne et al., J. Appl. Phys. 66 (1989) 1051-1056.

[4] S. Uemura and K. Torizuka, Jpn. J. Appl. Phys. 39 (2000) 3472-3473.

[5] R. P. Prasankumar et al., Opt. Express 11 (2003) 1265-1269.

[6] H. Castillo-Matadamas and R. Quintero-Torres, Laser Physics 18 (3) (2008) 246-252.

[7] U. Demirbas et al., Opt. Lett. 33 (2008) 59-592.

[8] A. Agnesi, F. Pirzio, E. Ugolotti, S. Y. Choi et al., Opt. Comm. 285 (2012) 742-745.

[9] U. Demirbas, S. Eggert, and A. Leitenstorfer, J. Opt. Soc. Am. B 29 (2012) 1894-1903.

[10] D. M. Kane and M. H. Dunn, Opt. Comm. 48 (1984) 295-300.

[11] K. K. Li, Appl. Opt. 21 (1982) 967-970.

[12] A. Agnesi, S. Dell'Acqua, G. C. Reali, P. G. Gobbi, and D. Ragazzi, Appl. Opt. 36(3) (1997) 597-601.

[13] D. M. Kane, App.Opt. 33(18) (1994) 3849-385.

[14] H. Kogelnik et al., IEEE J. Quant. Electron. QE-8(3) (1972) 373-379; H. Kogenik and T. Li, Laser beam and Resonators, Appl. Opt. 5 (1966) 1550-1567.

[15] D. C. Hanna, IEEE J. Quant. Electron. QE-5 (10) (1969) 483-488.

[16] . X. Wang, G. Hu, Y. Li and J. Yao, J. Opt. Soc. Am. A 11 (8) (1994) 2265-2270, doi: 10.1364/JOSAA.11.002265

[17] Jenkins, F.A. and H.E. White, Fundamentals of Optics, McGraw-Hill Book Company, 1976.

[18] D. Findlay and R. A. Clay, Phys. Lett. 20 (1966) 277-278. 